\title{
GI-graphs: a new class of graphs with many symmetries
}

\author{
Marston D.E. Conder • Tomaž Pisanski • \\ Arjana Žitnik
}

Received: 4 September 2012 / Accepted: 8 October 2013 / Published online: 30 October 2013

(C) Springer Science+Business Media New York 2013

\begin{abstract}
The class of generalized Petersen graphs was introduced by Coxeter in the 1950s. Frucht, Graver and Watkins determined the automorphism groups of generalized Petersen graphs in 1971, and much later, Nedela and Škoviera and (independently) Lovrečič-Saražin characterised those which are Cayley graphs. In this paper we extend the class of generalized Petersen graphs to a class of GI-graphs. For any positive integer $n$ and any sequence $j_{0}, j_{1}, \ldots, j_{t-1}$ of integers $\bmod n$, the $G I$-graph $G I\left(n ; j_{0}, j_{1}, \ldots, j_{t-1}\right)$ is a $(t+1)$-valent graph on the vertex set $\mathbb{Z}_{t} \times \mathbb{Z}_{n}$, with edges of two kinds:

- an edge from $(s, v)$ to $\left(s^{\prime}, v\right)$, for all distinct $s, s^{\prime} \in \mathbb{Z}_{t}$ and all $v \in \mathbb{Z}_{n}$,

- edges from $(s, v)$ to $\left(s, v+j_{s}\right)$ and $\left(s, v-j_{s}\right)$, for all $s \in \mathbb{Z}_{t}$ and $v \in \mathbb{Z}_{n}$.

By classifying different kinds of automorphisms, we describe the automorphism group of each $G I$-graph, and determine which $G I$-graphs are vertex-transitive and which are Cayley graphs. A GI-graph can be edge-transitive only when $t \leq 3$, or equivalently, for valence at most 4 . We present a unit-distance drawing of a remarkable $G I(7 ; 1,2,3)$.
\end{abstract}

M.D.E. Conder (凶)

Department of Mathematics, University of Auckland, Private Bag 92019, Auckland 1142,

New Zealand

e-mail: m.conder@auckland.ac.nz

T. Pisanski · A. Žitnik

Faculty of Mathematics and Physics, University of Ljubljana, Jadranska 19, 1000 Ljubljana, Slovenia

T. Pisanski

e-mail: Tomaz.Pisanski@fmf.uni-lj.si

A. Žitnik

e-mail: Arjana.Zitnik@fmf.uni-lj.si 
Keywords GI-graph · Generalized Petersen graph · Vertex-transitive graph · Edge-transitive graph · Circulant graph · Automorphism group · Wreath product . Unit-distance graph

\section{Introduction}

Trivalent graphs (also known as cubic graphs) form an extensively studied class of graphs. Among them, the Petersen graph is one of the most important finite graphs, constructible in many ways, and is a minimal counter-example for many conjectures in graph theory. The Petersen graph is the initial member of a family of graphs $G(n, k)$, known today as Generalized Petersen graphs, which have similar constructions. Generalized Petersen graphs were first introduced by Coxeter [3] in 1950, and were named in 1969 by Watkins [21].

A standard visualization of a generalized Petersen graph consists of two types of vertices: half of them belong to an outer rim, and the other half belong to an inner rim; and there are three types of edges: those in the outer rim, those in the inner rim, and the 'spokes', which form a 1-factor between the inner rim and the outer rim. The outer rim is always a cycle, while the inner rim may consist of several isomorphic cycles. A generalized Petersen graph $G(n, k)$ is given by two parameters $n$ and $k$, where $n$ is the number of vertices in each rim, and $k$ is the 'span' of the inner rim (which is the distance on the outer rim between the neighbours of two adjacent vertices on the inner rim).

The family $G(n, k)$ contains some very important graphs. Among others of particular interest are the $n$-prism $G(n, 1)$, the Dürer graph $G(6,2)$, the Möbius-Kantor graph $G(8,3)$, the dodecahedron $G(10,2)$, the Desargues graph $G(10,3)$, the Nauru graph $G(12,5)$, and of course the Petersen graph itself, which is $G(5,2)$.

Generalized Petersen graphs possess a number of interesting properties. For example, $G(n, k)$ is vertex-transitive if and only if either $n=10$ and $k=2$, or $k^{2} \equiv \pm 1$ $\bmod n$ [6], and a Cayley graph if and only if $k^{2} \equiv 1 \bmod n[14,18]$, and arctransitive only in the following seven cases: $(n, k)=(4,1),(5,2),(8,3),(10,2)$, $(10,3),(12,5)$ or $(24,5)[6]$.

If we want to maintain the symmetry between the two rims, then another parameter has to be introduced, allowing the span on the outer rim to be different from 1 . This gives the definition of an I-graph.

The family of $I$-graphs was introduced in 1988 in the Foster Census [4]. For some time this family failed to attract the attention of many researchers, possibly due to the fact that among all $I$-graphs, the only ones that are vertex-transitive are the generalized Petersen graphs $[1,15]$. Still, necessary and sufficient conditions for testing whether or not two $I$-graphs are isomorphic were determined in $[1,11]$, and these were used to enumerate all $I$-graphs in [19]. Also in [11] it was shown that all generalized Petersen graphs are unit-distance graphs, by representing them as isomorphic $I$-graphs. Furthermore, in [1] it was shown that the automorphism group of a connected $I$-graph $I(n, j, k)$ that is not a generalized Petersen graph is either dihedral or a group with presentation

$$
\Gamma=\left\langle\rho, \tau, \varphi \mid \rho^{n}=\tau^{2}=\varphi^{2}=1, \rho \tau \rho=\tau, \varphi \tau \varphi=\tau, \varphi \rho \varphi=\rho^{a}\right\rangle
$$


for some $a \in \mathbb{Z}_{n}$, and that among all $I$-graphs, only the generalized Petersen graphs can be vertex-transitive or edge-transitive.

In this paper we further generalize both of these families of graphs, and call them generalized I-graphs, or simply GI-graphs. We determine the group of automorphisms of any GI-graph. Moreover, we completely characterize the edge-transitive, vertex-transitive and Cayley graphs, among the class of $G I$-graphs.

At the end of the paper we briefly discuss the problem of unit-distance realizations of $G I$-graphs. This problem has been solved for $I$-graphs in [10]. We found a remarkable new example of a 4-valent unit-distance graph, namely $G I(7 ; 1,2,3)$, which is a Cayley graph on 21 vertices for the group $\mathbb{Z}_{7} \rtimes \mathbb{Z}_{3}$.

Let us note that ours is not the only possible generalization. For instance, see [13] for another approach, which is not much different from ours. The basic difference is that our approach uses complete graphs, while the approach by Lovrečič-Saražin, Pacco and Previtali in [13] uses cycles; their construction coincides with ours for $t \leq 3$, but not for larger $t$.

We acknowledge the use of MAGMA [2] in constructing and analysing examples of $G I$-graphs, and helping us to see patterns and test conjectures that led to many of the observations made and proved in this paper.

\section{Definition of $G I$-graphs and their properties}

For positive integers $n$ and $t$ with $n \geq 3$, let $J=\left(j_{0}, j_{1}, \ldots, j_{t-1}\right)$ be any sequence of integers such that $0<j_{k}<n$ and $j_{k} \neq n / 2$, for $0 \leq k<t$.

Then we define $G I(n ; J)$, or alternatively $G I\left(n ; j_{0}, j_{1}, \ldots, j_{t-1}\right)$, to be the graph with vertex set $\mathbb{Z}_{t} \times \mathbb{Z}_{n}$, and with edges of two types:

(a) an edge from $(s, v)$ to $\left(s^{\prime}, v\right)$, for all distinct $s, s^{\prime} \in \mathbb{Z}_{t}$ and all $v \in \mathbb{Z}_{n}$,

(b) edges from $(s, v)$ to $\left(s, v+j_{s}\right)$ and $\left(s, v-j_{s}\right)$, for all $s \in \mathbb{Z}_{t}$ and all $v \in \mathbb{Z}_{n}$.

This definition gives us an infinite family of graphs, which we call GI-graphs. The edges of type (a) are called the spoke edges, while those of type (b) are called the layer edges. Each vertex $(s, v)$ is adjacent to two layer edges since $j_{k} \neq n / 2$, for $0 \leq k<t$. It follows that the graph $G I\left(n ; j_{0}, j_{1}, \ldots, j_{t-1}\right)$ has $n t$ vertices, and is regular of valence $(t-1)+2=t+1$. (Note that it would not be regular if we allowed $n / 2$ to be an element of $J$ when $|J|>1$.) Also for each $s \in \mathbb{Z}_{t}$ the set $L_{s}=\{(s, v)$ : $\left.v \in \mathbb{Z}_{n}\right\}$ is called a layer, and for each $v \in \mathbb{Z}_{n}$ the set $S_{v}=\left\{(s, v): s \in \mathbb{Z}_{t}\right\}$ is called a spoke. We observe that the induced subgraph on each spoke is a complete graph $K_{t}$ of order $t$. On the other hand, the induced subgraph on the layer $L_{s}$ is a union of $d_{s}$ cycles of length $n / d_{s}$, where $d_{s}=\operatorname{gcd}\left(n, j_{s}\right)$.

In the case $t=1$, the graph $G I\left(n ; j_{0}\right)$ is simply a union of disjoint isomorphic cycles of length $n / \operatorname{gcd}\left(n, j_{0}\right)$. In the case $t=2$, we have $I$-graphs; for example, $G I(n ; 1, j)$ is a generalized Petersen graph, for every $j$, and in particular, $G I(10 ; 1,2)$ is the dodecahedral graph (the 1-skeleton of a dodecahedron). Some other examples are illustrated in Fig. 1.

Note that taking $j_{k}^{\prime}= \pm j_{k}$ for all $k$ gives a $G I$-graph $G I\left(n ; j_{0}^{\prime}, j_{1}^{\prime}, \ldots, j_{t-1}^{\prime}\right)$ that is exactly the same as $G I\left(n ; j_{0}, j_{1}, \ldots, j_{t-1}\right)$. Similarly, any permutation of 


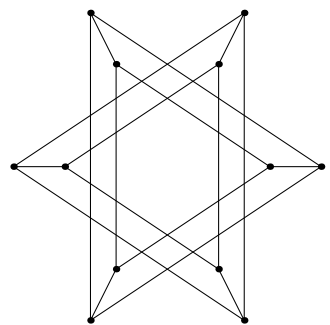

(a)

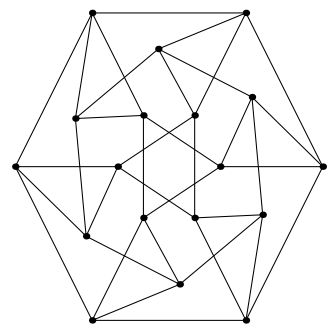

(b)

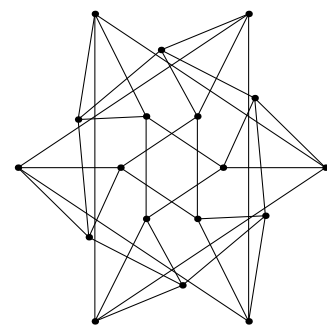

(c)

Fig. $1 G I$-graphs $G I(6 ; 2,2), G I(6 ; 1,1,2)$, and $G I(6 ; 2,1,2)$

$j_{0}, j_{1}, \ldots, j_{t-1}$ gives a $G I$-graph isomorphic to $G I\left(n ; j_{0}, j_{1}, \ldots, j_{t-1}\right)$. Therefore we will usually assume that $0<j_{k}<n / 2$ for all $k$, and that $j_{0} \leq j_{1} \leq \cdots \leq j_{t-1}$. In this case, we say that the $G I$-graph $G I\left(n ; j_{0}, j_{1}, \ldots, j_{t-1}\right)$ is in standard form.

The following gives a partial answer to the problem of distinguishing between two GI-graphs.

Proposition 1 Suppose $j_{0}, j_{1}, \ldots, j_{t-1} \neq 0$ or $n / 2$ modulo $n$, and a is a unit in $\mathbb{Z}_{n}$. Then the graph $G I\left(n ; a j_{0}, a j_{1}, \ldots, a j_{t-1}\right)$ is isomorphic to the graph $G I\left(n ; j_{0}, j_{1}\right.$, $\left.\ldots, j_{t-1}\right)$.

Proof Since $a$ is coprime to $n$, the numbers $a v$ for $0 \leq v<n$, are all distinct in $\mathbb{Z}_{n}$, and so we can label the vertices of $G I\left(n ; a j_{0}, a j_{1}, \ldots, a j_{t-1}\right)$ as ordered pairs $(s, a v)$ for $s \in \mathbb{Z}_{t}$ and $v \in \mathbb{Z}_{n}$. Now define a mapping $\varphi: V\left(G I\left(n ; a j_{0}, a j_{1}, \ldots, a j_{t-1}\right)\right) \rightarrow$ $V\left(G I\left(n ; j_{0}, j_{1}, \ldots, j_{t-1}\right)\right)$ by setting $\varphi((s, a v))=(s, v)$ for all $s \in \mathbb{Z}_{t}$ and all $v \in \mathbb{Z}_{n}$. This is clearly a bijection, and since a vertex $(s, a v)$ in $G I\left(n ; a j_{0}, a j_{1}, \ldots, a j_{t-1}\right)$ is adjacent to $\left(s^{\prime}, a v\right)$ for each $s^{\prime} \in \mathbb{Z}_{t} \backslash\{s\}$ and to $\left(s, a v \pm a j_{s}\right)=\left(s, a\left(v \pm j_{s}\right)\right)$, it is easy to see that $\varphi$ is also a graph homomorphism.

We may view the sequence $J$ as a multiset. We will say that $J$ is in canonical form if it is lexicographically first among all the sequences (or ordered multisets) that give isomorphic copies of $G I(n ; J)$ via Proposition 1 .

We now list some other properties of GI-graphs. Proposition 3 shows that the spoke edges are easy to recognise when $t>3$.

Proposition 2 The graph $G I\left(n ; j_{0}, j_{1}, \ldots, j_{t-1}\right)$ admits a factorization into a $(t-1)$ factor $n K_{t}$ and a 2-factor (namely the spokes and the layers).

Proposition 3 An edge of a GI-graph with 4 or more layers is a spoke edge if and only if it belongs to some clique of size 4.

Proof No edge between two vertices in the same layer can lie in a $K_{4}$ subgraph, because the subgraph induced on each layer is a union of cycles, and no two spokes between two different layers can have a common vertex. 
Proposition 4 Let $d=\operatorname{gcd}\left(n, j_{0}, j_{1}, \ldots, j_{t-1}\right)$. Then the graph $G I\left(n ; j_{0}, j_{1}, \ldots\right.$, $\left.j_{t-1}\right)$ is a disjoint union of $d$ copies of $G I\left(n / d ; j_{0} / d, j_{1} / d, \ldots, j_{t-1} / d\right)$. In particular, the graph $G I\left(n ; j_{0}, j_{1}, \ldots, j_{t-1}\right)$ is connected if and only if $d=1$.

Proof First observe that the edges of every spoke make up a clique (of order $t$ ), so the graph is connected if and only if every two spokes are connected via the layer edges. Now there exists an edge between two spokes $S_{u}$ and $S_{v}$ whenever $v-u= \pm j_{s}$ for some $s$, and hence a path of length 2 between $S_{u}$ and $S_{v}$ whenever $v-u$ is a $\mathbb{Z}_{n}$-linear combination of some $j_{s}$ and $j_{s^{\prime}}$, and so on. Thus $S_{u}$ and $S_{v}$ lie in the same connected component of the graph if and only if $v-u$ is expressible $(\bmod n)$ as a $\mathbb{Z}$-linear combination of $j_{0}, j_{1}, \ldots, j_{t-1}$, say $v-u=c n+c_{0} j_{0}+c_{1} j_{1}+\cdots+c_{t-1} j_{t-1}$ for some $c_{0}, c_{1}, \ldots, c_{t-1} \in \mathbb{Z}$. By Bezout's identity, this occurs if and only if $v-u$ is a multiple of $\operatorname{gcd}\left(n, j_{0}, j_{1}, \ldots, j_{t-1}\right)=d$. It follows that the graph has $d$ components, each containing a set of spokes $S_{v}$ with $v=u+j d$ for fixed $u$ and variable $j$, that is, with subscripts differing by multiples of $d$. Finally, since $(v-u) / d=c(n / d)+$ $c_{0}\left(j_{0} / d\right)+c_{1}\left(j_{1} / d\right)+\cdots+c_{t-1}\left(j_{t-1} / d\right)$, it is easy to see that each component is isomorphic to $G I\left(n / d ; j_{0} / d, j_{1} / d, \ldots, j_{t-1} / d\right)$.

Finally, note that the restriction of a $G I$-graph to any proper subset of its layers gives rise to another $G I$-graph. In particular, if $J$ and $K$ are multisets with $J \subseteq K$, then $G I(n ; J)$ is an induced subgraph of $G I(n ; K)$.

\section{Automorphisms of $G I$-graphs}

In this section, we consider the possible automorphisms of a $G I$-graph $X=G I(n ; J)$, where $J=\left\{j_{0}, j_{1}, \ldots, j_{t-1}\right\}$ is any multiset. If $X$ is disconnected, then since all connected components of $X$ are isomorphic to each other (by Proposition 4), we may simply reduce this to the consideration of automorphisms of a connected component of $X$ (and then find the automorphism group using a theorem of Frucht [5], cf. [8]). Hence from now on, we will assume that $X$ is connected.

The set of edges of $X=G I(n ; J)$ may be partitioned into spoke edges and layer edges, and we will call this partition of edges the fundamental edge-partition of $X$. We know that the graph induced on the spoke edges is a collection of complete graphs, and that the graph induced on the layer edges is a collection of cycles (with each cycle belonging to a single layer, but with a layer being composed of two or more cycles of the same length $n / \operatorname{gcd}\left(n, j_{s}\right)$ if the corresponding element $j_{s}$ of $J$ is not a unit $\bmod n$ ).

We will say that an automorphism of $X$ respects the fundamental edge-partition if it takes spoke edges to spoke edges, and layer edges to layer edges. Any automorphism of $X$ that does not respect the fundamental edge-partition (and so takes some layer edge to a spoke edge, and some spoke edge to a layer edge) will be called skew.

Theorem 5 Let $X$ be a connected GI-graph with $t$ layers, where $t \geq 2$. If $X$ has a skew automorphism, then either $t=2$ and $X$ is isomorphic to one of the seven special generalized Petersen graphs $G(4,1), G(5,2), G(8,3), G(10,2), G(10,3)$, 
$G(12,5)$ and $G(24,5)$, or $t=3$ and $X$ is isomorphic to $G I(3 ; 1,1,1)$. Moreover, each of these eight graphs is arc-transitive (and is therefore both vertex-transitive and edge-transitive).

Proof First, if $t>3$ then no layer edge lies in a clique of size $t$, but every spoke edge does, and therefore no automorphism can map a spoke edge to a layer edge. Thus $t \leq 3$.

Next, suppose $t=3$, and let $\varphi$ be an automorphism taking an edge $e$ of some spoke $S_{v}$ to an edge $e^{\prime}$ of some layer $L_{s}$. Since every edge of a spoke lies in a triangle, namely the spoke itself, it follows that $\varphi$ must take the whole spoke $S_{v}=\{(0, v),(1, v),(2, v)\}$ containing $e$ to some triangle containing the layer edge $e^{\prime}$, and then the other two edges of the triangle $\{\varphi(0, v), \varphi(1, v), \varphi(2, v)\}$ must be edges from the same layer as $e^{\prime}$, namely $L_{s}$. It follows that $j_{s}=n / 3$. But then since each of the images $\varphi(0, v), \varphi(1, v), \varphi(2, v)$ lies in two triangles (namely a spoke and a triangle in $\left.L_{s}\right)$, each of the vertices $(0, v),(1, v)$ and $(2, v)$ must similarly lie in two triangles, and it follows that all three layers contain a triangle, so $j_{0}=j_{1}=j_{2}=n / 3$. In particular, $\operatorname{gcd}\left(j_{0}, j_{1}, j_{2}\right)=n / 3$, and by connectedness, Proposition 4 implies $n / 3=1$, so $n=3$ and $j_{0}=j_{1}=j_{2}=1$. Thus $X$ is $G I(3 ; 1,1,1)$, which is wellknown to be arc-transitive (see [13], for example).

Finally, for the case $t=2$, everything we need was proved in [6] and [1].

Corollary 6 Every edge-transitive connected GI-graph with more than one layer is isomorphic to one of the eight graphs listed in Theorem 5.

Hence from now on, we will consider only the automorphisms that respect the fundamental edge-partition. There are three special classes of such automorphisms:

(1) automorphisms that preserve every layer

(2) automorphisms that preserve every spoke

(3) automorphisms that permute both the layers and the spokes non-trivially.

We will consider particular cases of automorphisms of these types below.

Define mappings $\rho: V(X) \rightarrow V(X)$ and $\tau: V(X) \rightarrow V(X)$ given by

$$
\rho(s, v)=(s, v+1) \quad \text { and } \quad \tau(s, v)=(s,-v) \quad \text { for all } s \in \mathbb{Z}_{t} \text { and all } v \in \mathbb{Z}_{n} .
$$

Clearly these are automorphisms of $X$ of type (1), permuting the vertices in each layer. Indeed $\rho$ can be viewed as a rotation (of order $n$ ), and $\tau$ as a reflection (of order 2), and it follows that the automorphism group of $X$ contains a dihedral subgroup of order $2 n$, generated by $\rho$ and $\tau$. These $2 n$ automorphisms are all of type (1), and all of them respect the fundamental edge-partition of $X$.

Next, if two of the members of the multiset $J$ are equal, say $j_{s_{1}}=j_{s_{2}}$ for $s_{1} \neq s_{2}$, then we have an automorphism $\lambda_{i, s_{1}, s_{2}}$ that exchanges two cycles of layers $L_{s_{1}}$ and $L_{s_{2}}$, but preserves every spoke. These automorphisms are of type (2). 
Proposition 7 Suppose $j_{s_{1}}=j_{s_{2}}$ where $s_{1} \neq s_{2}$, and define $d=\operatorname{gcd}\left(n, j_{s_{1}}\right)=$ $\operatorname{gcd}\left(n, j_{s_{2}}\right)$. Then for each $i \in \mathbb{Z}_{d}$, the mapping $\lambda_{i, s_{1}, s_{2}}: V(X) \rightarrow V(X)$ given by

$$
\lambda_{i, s_{1}, s_{2}}(s, v)= \begin{cases}\left(s_{2}, v\right) & \text { if } s=s_{1} \text { and } v \equiv i \bmod d \\ \left(s_{1}, v\right) & \text { if } s=s_{2} \text { and } v \equiv i \bmod d \\ (s, v) & \text { otherwise }\end{cases}
$$

is an automorphism of $X$, which respects the fundamental edge-partition, and preserves all layers other than $L_{s_{1}}$ and $L_{s_{2}}$.

Proof This is obviously a permutation of $V(X)$, preserving adjacency. Moreover, it is also clear that $\lambda_{i, s_{1}, s_{2}}$ preserves every spoke $S_{v}$, and exchanges one of the cycles in layer $L_{S_{1}}$ with the corresponding cycle in layer $L_{S_{2}}$, while preserving all other layer cycles.

Corollary 8 Suppose $j_{s_{1}}=j_{s_{2}}$ where $s_{1} \neq s_{2}$, and define $d=\operatorname{gcd}\left(n, j_{s_{1}}\right)=$ $\operatorname{gcd}\left(n, j_{s_{2}}\right)$. Then the product

$$
\lambda_{s_{1}, s_{2}}:=\lambda_{0, s_{1}, s_{2}} \lambda_{1, s_{1}, s_{2}} \cdots \lambda_{d-1, s_{1}, s_{2}}
$$

is an automorphism of $X$ that respects the fundamental edge-partition, and exchanges layers $L_{s_{1}}$ and $L_{s_{2}}$, while preserving every other layer.

There is another family of automorphisms exchanging layers that exist in some situations; but these automorphisms do not preserve spokes, and so they are of type (3):

Proposition 9 Let a be a unit in $\mathbb{Z}_{n}$ with the property that aJ $=\left\{p_{0} j_{0}, p_{1} j_{1}, \ldots\right.$, $\left.p_{t-1} j_{t-1}\right\}$ where $p_{i} \in\{-1,1\}$ for all $i$, and then let $\alpha: \mathbb{Z}_{t} \rightarrow \mathbb{Z}_{t}$ be a bijection with the property that $j_{\alpha(s)} \in\left\{-a j_{s}, a j_{s}\right\}$ for all $s \in \mathbb{Z}_{t}$. Then the mapping $\sigma_{a}: V(X) \rightarrow$ $V(X)$ given by

$$
\sigma_{a}(s, v)=(\alpha(s), a v) \quad \text { for all } s \in \mathbb{Z}_{t} \text { and all } v \in \mathbb{Z}_{n}
$$

is an automorphism of $X$ that respects the fundamental edge-partition.

Remarks Note that the mapping $\alpha$ is not uniquely determined if there exist distinct $s_{1}$ and $s_{2}$ for which $j_{s_{1}}= \pm j_{s_{2}}$, but we can always define the mapping $\alpha$ so that it is a bijection (and satisfies $j_{\alpha(s)}= \pm a j_{s}$ for all $s \in \mathbb{Z}_{t}$ ). Indeed $\alpha$ is uniquely determined if we require that $\alpha\left(s_{1}\right)<\alpha\left(s_{2}\right)$ whenever $s_{1}<s_{2}$ and $j_{s_{1}}= \pm j_{s_{2}}$.

On the other hand, $\sigma_{a}$ is not defined when the condition $a J=\left\{p_{0} j_{0}, p_{1} j_{1}, \ldots\right.$, $\left.p_{t-1} j_{t-1}\right\}$ fails (or equivalently, when $a(J \cup-J) \neq J \cup-J$ ). Note also that $\sigma_{1}$ is the identity automorphism, while $\sigma_{-1}$ is the automorphism $\tau$ defined earlier, since for $a=-1$ we may take $\alpha$ as the identity permutation and then $\sigma_{-1}(s, v)=(s,-v)=$ $\tau(s, v)$ for every vertex $(s, v)$.

Proof First, let $b$ be the multiplicative inverse of $a$ in $\mathbb{Z}_{n}^{*}$. Then for any $(s, v) \in$ $\mathbb{Z}_{t} \times \mathbb{Z}_{n}$, we have $\sigma_{a}\left(\alpha^{-1}(s), b v\right)=\left(\alpha\left(\alpha^{-1}(s)\right), a b v\right)=(s, v)$, and therefore $\sigma_{a}$ is 
surjective. Since $V(X)$ is finite, it follows that $\sigma_{a}$ is a permutation. Also $\sigma_{a}$ preserves edges, indeed it respects the fundamental edge-partition, because it takes each neighbour $\left(s^{\prime}, v\right)$ of the vertex $(s, v)$ in the spoke $S_{v}$ to the neighbour $\left(\alpha\left(s^{\prime}\right), a v\right)$ of the vertex $(\alpha(s), a v)$ in the spoke $S_{a v}$, and takes the two neighbours $\left(s, v \pm j_{s}\right)$ of the vertex $(s, v)$ in the layer $L_{s}$ to the two neighbours $\sigma_{a}\left(s, v \pm j_{s}\right)=\left(\alpha(s), a\left(v \pm j_{s}\right)\right)=$ $\left(\alpha(s), a v \pm a j_{s}\right)=\left(\alpha(s), a v \pm j_{\alpha(s)}\right)$ of the vertex $(\alpha(s), a v)$ in the layer $L_{\alpha(s)}$.

In the remaining part of this section we will show that if the $G I$-graph $X$ is connected, then the automorphisms described above and their products give all of the automorphisms of $X$ that respect the fundamental edge-partition.

For this we require two technical lemmas, the proofs of which are obvious.

Lemma 10 Let $X$ be a connected GI-graph with at least two layers. Then every automorphism of $X$ that preserves spoke edges must permute the spokes (like blocks of imprimitivity).

Lemma 11 Every automorphism of a GI-graph that respects the fundamental edgepartition must permute the layer cycles.

It will also be helpful to relate the automorphisms of a $G I$-graph to the automorphisms of the corresponding circulant graph.

Let $S$ be a subset of $\mathbb{Z}_{n}$ such that $S=-S$ and $0 \notin S$. Then the circulant graph $\operatorname{Circ}(n ; S)$ is defined as the graph with vertex set $\mathbb{Z}_{n}$, such that vertices $u$ and $v$ are adjacent precisely when $u-v \equiv a \bmod n$ for some $a \in S$. Equivalently, this is the Cayley graph for $\mathbb{Z}_{n}$ given by the subset $S$. Note that $\operatorname{Circ}(n ; S)$ is connected if and only if $S$ additively generates $\mathbb{Z}_{n}$, that is, if and only if some linear combination of the members of $S$ is $1 \bmod n$.

Now suppose that $S=\left\{s_{1}, \ldots, s_{c}\right\}$, and that $\Gamma=\operatorname{Circ}(n ; S)$ is connected.

For $1 \leq i \leq c$, let $G_{i, 1}, G_{i, 2}, \ldots, G_{i, k_{i}}$ be the distinct cosets of the cyclic subgroup $G_{i, 1}=\left\langle s_{i}\right\rangle$ in $G=\langle S\rangle$. Then we can form a partition $\mathcal{C}=\left\{C_{i j}\right\}$ of the edges of $\Gamma$, where

$$
C_{i j}=\left\{\left\{g, g+s_{i}\right\}: g \in G_{i, j}\right\} \quad \text { for } 1 \leq j \leq k_{i} \text { and } 1 \leq i \leq c .
$$

Notice that each part $C_{i j}$ of $\mathcal{C}$ consists of precisely the edges of a cycle formed by adding multiples of the single element $s_{i}$ of $S$ to a member of the coset $G_{i, j}$.

We say that an automorphism $\varphi$ of $\Gamma$ respects the partition $\mathcal{C}$ if $\varphi\left(C_{i j}\right) \in \mathcal{C}$ for every $C_{i j} \in \mathcal{C}$. We have the following, thanks to Joy Morris.

Theorem 12 Suppose the circulant graph $\Gamma=\operatorname{Circ}(n ; S)$ is connected. If $\psi$ is an automorphism of $\Gamma$ which fixes the vertex 0 and respects the partition $\mathcal{C}=\left\{C_{i j}\right\}$, then $\psi$ is induced by some automorphism of $\mathbb{Z}_{n}$-that is, there exists a unit a $\in \mathbb{Z}_{n}$ with the property that $\psi(x)=$ ax for every $x \in \mathbb{Z}_{n}$ (and in particular, aS $=S$ ).

For a proof (by induction on $|S|$ ), see [17]. To apply it, we associate with our graph $X=G I(n ; J)$ the circulant graph $Y=\operatorname{Circ}(n ; S \cup-S)$, where $S$ is the underlying set of $J$. 
Note that the projection $\eta: V(X) \rightarrow V(Y)$ given by $\eta(s, v)=v$ takes every layer edge $\left\{(s, v),\left(s, v+j_{s}\right)\right\}$ of $X$ to the edge $\left\{v, v+j_{s}\right\}$ of $Y$, and hence gives a graph homomorphism from the subgraph of $X$ induced on layer edges onto the graph $Y$.

Proposition 13 Every automorphism of $X=G I(n ; J)$ that preserves the set of spoke edges induces an automorphism of $Y=\operatorname{Circ}(n ; S \cup-S)$ that respects the partition $\mathcal{C}=\left\{C_{i j}\right\}$.

Proof Any such automorphism $\varphi$ induces a permutation on the set of spokes of $X$, and hence under the above projection $\eta$, induces an automorphism of $Y$, say $\psi$. Moreover, since $\varphi$ preserves the layer edges, it must permute the layer cycles among themselves, and it follows that $\psi$ respects the partition $\mathcal{C}=\left\{C_{i j}\right\}$.

Corollary 14 Suppose $X$ is connected. Then every automorphism of $X=G I(n ; J)$ that respects the fundamental edge-partition of $X$ is expressible as a product of powers of the rotation $\rho$, the reflection $\tau$, and the automorphisms $\lambda_{i, s_{1}, s_{2}}$ and $\sigma_{a}$ defined in Propositions 7 and 9.

Proof First, any such automorphism $\varphi$ induces a permutation on the set of spokes of $X$, and so by multiplying by a suitable element of the dihedral group of order $2 n$ generated by $\rho$ and $\tau$, we may replace $\varphi$ by an automorphism $\varphi^{\prime}$ that respects the fundamental edge-partition of $X$, and preserves the spoke $S_{0}$. In particular, $\varphi^{\prime}$ induces an automorphism of $Y=\operatorname{Circ}(n ; S \cup-S)$ that fixes the vertex 0 . By Theorem 12, this automorphism of $Y$ is induced by multiplication by some unit $a \in \mathbb{Z}_{n}$, and then by multiplying by the inverse of $\sigma_{a}$ we may replace $\varphi^{\prime}$ by an automorphism $\varphi^{\prime \prime}$ that preserves all of the spokes $S_{v}$. Finally, since $\varphi^{\prime \prime}$ preserves all of the spokes and also permutes the layer cycles among themselves, $\varphi^{\prime \prime}$ is expressible as a product of the automorphisms $\lambda_{i, s_{1}, s_{2}}$ defined in Proposition 7.

As a special case, we have also the following, for the automorphisms that preserve layers:

Corollary 15 Suppose $X$ is connected. Then any automorphism of $X=G I(n ; J)$ that takes layers to layers is a product of powers of the rotation $\rho$, the reflection $\tau$, and the automorphisms $\lambda_{s_{1}, s_{2}}$ and $\sigma_{a}$ defined in Corollary 8 and Proposition 9.

\section{Automorphism groups of GI-graphs}

Now that we know all possible automorphisms of a GI-graph, it is not difficult to determine their number, and construct the automorphism groups in many cases. We will sometimes use $F(n ; J)$ to denote the number of automorphisms of $G I(n ; J)$, and $A(n ; J)$ to denote the automorphism group $G I(n ; J)$.

The automorphism group $A(n ; J)$ of $G I(n ; J)$ always contains a dihedral subgroup of order $2 n$, generated by the rotation $\rho$ and the reflection $\tau$, defined in ( $\dagger$ ) in the previous section (before Proposition 7). Note that the relations $\rho^{n}=\tau^{2}=$ $(\rho \tau)^{2}=1$ hold, with the third of these being equivalent to $\tau \rho \tau=\rho^{-1}$.

We split the consideration of $F(n ; J)$ and $A(n ; J)$ into four cases, below. 


\subsection{The disconnected case}

Let $d=\operatorname{gcd}(n, J)$. Then $G I(n ; J)$ is the disjoint union of $d$ isomorphic copies of $G I(n ; J / d)$, by Proposition 4. This reduces the computation of $\operatorname{Aut}(X)$ to the case of connected $G I$-graphs. In particular, we have

$$
A(n ; J) \cong A(n ; J / d)>\operatorname{Sym}(d)
$$

so $\operatorname{Aut}(G I(n ; J))$ is the wreath product of $\operatorname{Aut}(G I(n ; J / d))$ by the symmetric group $\operatorname{Sym}(d)$ of degree $d$, and therefore

$$
F(n ; J)=|\operatorname{Aut}(G I(n ; J))|=d !(F(n ; J / d))^{d} .
$$

\subsection{The edge-transitive case}

The eight connected edge-transitive $G I$-graphs with more than one layer were given in Theorem 5. Seven of them are generalized Petersen graphs, with $J=\{1, k\}$ for some $k \in \mathbb{Z}_{n}^{*}$, and their automorphism groups are known-see [6] or [13] for example.

For each of these seven graphs, all of which are cubic, there is an automorphism $\mu$ of order 3 that fixes the vertex $(0,0)$ and induces a 3 -cycle on its neighbours $(1,0),(0,1)$ and $(0, n-1)$. In particular, this automorphism $\mu$ takes the spoke edge $\{(0,0),(1,0)\}$ to the layer edge $\{(0,0),(0,1)\}$, and its effect on the other vertices is easily determined.

In the cases $(n, k)=(4,1),(8,3),(12,5)$ and $(24,5)$, where $n \equiv 0 \bmod 4$ and $k^{2} \equiv 1 \bmod n$, the three automorphisms $\rho, \tau$ and $\mu$ generate $A(n ; J)$ and satisfy the defining relations

$$
\rho^{n}=\tau^{2}=\mu^{3}=(\rho \tau)^{2}=(\rho \mu)^{2}=(\tau \mu)^{2}=\left[\rho^{4}, \mu\right]=1
$$

for a group of order $12 n$ which we may denote for the time being as $\Gamma(n, k)$, although strictly speaking, the second parameter $k$ is not necessary.

Similarly in the case $(n, k)=(10,2)$, the three automorphisms $\rho, \tau$ and $\mu$ generate $A(n ; J)$, which has order $12 n$, but they satisfy different defining relations, with the relation $\left[\rho^{4}, \mu\right]=1$ replaced by $\mu \rho^{-1} \mu \rho^{2} \mu^{-1} \rho^{2} \tau=1$. In the other two cases (namely $(n, k)=(5,2)$ and $(10,3))$, the automorphisms $\rho, \tau$ and $\mu$ generate a subgroup of index 2 in $A(n ; J)$, which has order $24 n$.

In summary, the automorphism groups of the eight connected edge-transitive $G I$ graphs with more than one layer, and their orders, can be described as below:

$$
\begin{array}{rlrl}
\operatorname{Aut}(G I(4 ; 1,1)) & \cong \Gamma(4,1) \cong S_{4} \times \mathbb{Z}_{2} & F(4 ; 1,1) & =48 \\
\operatorname{Aut}(G I(5 ; 1,2)) & \cong S_{5} & F(5 ; 1,2) & =120 \\
\operatorname{Aut}(G I(8 ; 1,3)) & \cong \Gamma(8,3) & F(8 ; 1,3) & =96 \\
\operatorname{Aut}(G I(10 ; 1,2)) & \cong A_{5} \times \mathbb{Z}_{2} & F(10 ; 1,2) & =120 \\
\operatorname{Aut}(G I(10 ; 1,3)) & \cong S_{5} \times \mathbb{Z}_{2} & F(10 ; 1,3) & =240 \\
\operatorname{Aut}(G I(12 ; 1,5)) & \cong \Gamma(12,5) & F(12 ; 1,5) & =144 \\
\operatorname{Aut}(G I(24 ; 1,5)) & \cong \Gamma(24,5) & F(24 ; 1,5) & =288 \\
\operatorname{Aut}(G I(3 ; 1,1,1)) & \cong\left(D_{6} \times D_{6}\right) \times \mathbb{Z}_{2} & F(3 ; 1,1,1) & =72 .
\end{array}
$$

See [6] and/or [13] for further details. 


\subsection{The case where $J$ is a set (with no repetitions)}

Suppose $J$ is a set (and not a multiset), in standard form, and let $X=G I(n ; J)$. If $X$ is not connected, then Sect. 4.1 applies, while if $X$ is connected and edge-transitive, then Sect. 4.2 applies, so we will suppose that $X$ is connected but not edge-transitive.

Then by Corollary 15, we know that the automorphism group of $X$ is generated by the automorphisms $\rho, \tau$ and the set $\left\{\sigma_{a}: a \in A\right\}$, where

$$
A=\left\{a \in \mathbb{Z}_{n}^{*} \mid a(J \cup-J)=J \cup-J\right\} .
$$

It is easy to see that $A$ is a subgroup of $\mathbb{Z}_{n}^{*}$. Indeed since $\sigma_{1}$ is trivial, $\sigma_{-1}=\tau$, and $\sigma_{a} \sigma_{b}=\sigma_{a b}$ for all $a, b \in A$, the set $S=\left\{\sigma_{a}: a \in A\right\}$ is a subgroup of $\operatorname{Aut}(X)$, isomorphic to $A$. In particular, $S$ is abelian. It is also easy to see that if composition of functions is read from left to right, and $\alpha$ is the bijection satisfying $j_{\alpha(s)}= \pm a j_{s}$ for all $s \in \mathbb{Z}_{t}$, then

$$
\begin{aligned}
\left(\rho \sigma_{a}\right)(s, v) & =\sigma_{a}(s, v+1)=(\alpha(s), a(v+1))=(\alpha(s), a v+a)=\rho^{a}(\alpha(s), a v) \\
& =\left(\sigma_{a} \rho^{a}\right)(s, v)
\end{aligned}
$$

for every vertex $(s, v)$, and so $\rho \sigma_{a}=\sigma_{a} \rho^{a}$ for all $a \in A$. Rearranging, we have $\sigma_{a}^{-1} \rho \sigma_{a}=\rho^{a}$ for all $a \in A$, which shows that every element of $S$ normalizes the cyclic subgroup of order $n$ generated by the rotation $\rho$. Finally, again since $\tau=\sigma_{-1} \in S$, this implies that the automorphism group of $X=G I(n ; J)$ is a semidirect product:

$$
A(n ; J)=\langle\{\rho\} \cup S\rangle \cong\langle\rho\rangle \rtimes S \cong C_{n} \rtimes A, \quad \text { of order } F(n ; J)=n|A| .
$$

\subsection{The general case}

In this subsection we deal with all remaining possibilities, in which $J$ is a multiset with repeated elements, in standard form, and $X=G I(n ; J)$ is connected but not edge-transitive. Here we need two new sets of parameters, namely the multiplicity $m_{j}$ in $J$ of each element $j$ from the underlying set of $J$ (that is, the number of $s \in \mathbb{Z}_{t}$ for which $\left.j_{s}=j\right)$, and $d_{j}=\operatorname{gcd}(n, j)$ for all such $j$.

Also we need the set $B$ of all $a \in \mathbb{Z}_{n}^{*}$ with the property that $a J=\left\{ \pm j_{0}, \pm j_{1}, \ldots\right.$, $\left.\pm j_{t-1}\right\}$. Note that this is always a subgroup of $\mathbb{Z}_{n}^{*}$, but is not always the same as the subgroup $A=\left\{a \in \mathbb{Z}_{n}^{*} \mid a(J \cup-J)=J \cup-J\right\}$ that we took in the previous subsection, since the multiplicities of $j$ and $\pm a j$ in $J$ might not be the same for some $a \in A$, but clearly they must be the same for every $a \in B$.

For example, consider the graph $G I(15 ; 3,5,5,5,6,6)$. Here $\left(m_{3}, m_{5}, m_{6}\right)=$ $(1,3,2),\left(d_{3}, d_{5}, d_{6}\right)=(3,5,3)$, and $B=\{1,4,11,14\}$ (while $A=\{1,2,4,7,8,11$, $13,14\})$.

Now by Corollary 14 we know that the automorphism group of $X$ is generated by the automorphisms $\rho$ and $\tau$, the automorphisms $\sigma_{a}$ for $a \in B$ (as defined in Proposition 9), and the automorphisms $\lambda_{i, s, s^{\prime}}$ (as defined in Proposition 7) that mix cycles.

Just as in the previous case, the set $S=\left\{\sigma_{a}: a \in B\right\}$ is a subgroup of $\operatorname{Aut}(X)$, isomorphic to the subgroup $B$ of $\mathbb{Z}_{n}^{*}$. Again also we have $\sigma_{a}^{-1} \rho \sigma_{a}=\rho^{a}$ for all $a \in B$, 
and so every element of $S$ normalizes the cyclic subgroup of order $n$ generated by the rotation $\rho$.

Next, for each $j \in J$, define $\Omega_{j}=\left\{s \in \mathbb{Z}_{t} \mid j_{s}=j\right\}$, which is a set of size $m_{j}$, and for the time being, let $d=d_{j}=\operatorname{gcd}(n, j)$. Also define $\Omega_{j, i}=\{(s, v) \in V(X) \mid$ $\left.s \in \Omega_{j}, v \equiv i \bmod d\right\}$, for $j \in J$ and $i \in \mathbb{Z}_{d}$ (where $\left.d=\operatorname{gcd}(n, j)\right)$. Note that $\left|\Omega_{j, i}\right|=$ $m_{j} n / d$, because $\Omega_{j, i}$ is like a strip of vertices across $m_{j}$ layers of $X$, containing the $n / d$ vertices of one cycle from each of these layers.

By Proposition 7, for every two distinct $s_{1}, s_{2}$ in $\Omega_{j}$ and every $i \in \mathbb{Z}_{d}$, there exists an involutory automorphism $\lambda_{i, s_{1}, s_{2}}$ that exchanges one of the $d$ cycles from layer $L_{S_{1}}$ with the corresponding cycle from layer $L_{s_{2}}$, and preserves every spoke.

For given $j \in J$, define $T_{j, i}$ to be the subgroup generated by the automorphisms $\lambda_{i, s_{1}, s_{2}}$ with $s_{1}, s_{2}$ in $\Omega_{j}$, for each $i \in \mathbb{Z}_{d}$ (where $d=\operatorname{gcd}(n, j)$ ), and let $T_{j}$ be the subgroup generated by all such $T_{j, i}$. Also let $N$ be the subgroup generated by all such $T_{j}$.

Each automorphism $\lambda_{i, s_{1}, s_{2}}$ induces a transposition on the set of $m_{j}$ layer cycles containing the vertices of the set $\Omega_{j, i}$. If we let the pair $\left\{s_{1}, s_{2}\right\}$ vary, we get all such transpositions, and hence for fixed $j$ and $i$, the subgroup $T_{j, i}$ is isomorphic to the symmetric group $\operatorname{Sym}\left(m_{j}\right)$, acting with $n / d$ orbits of length $m_{j}$ on $\Omega_{j, i}$ and fixing all other vertices.

Moreover, for any two distinct $i_{1}, i_{2}$ in $\mathbb{Z}_{d}$, the elements of $T_{j, i_{1}}$ and $T_{j, i_{2}}$ move disjoint sets of vertices (namely $\Omega_{j, i_{1}}$ and $\Omega_{j, i_{2}}$ ), and hence commute with each other. Hence the subgroup $T_{j}$ (generated by all the $T_{j, i}$, for given $j$ ) is isomorphic to the direct product of $d$ copies of $\operatorname{Sym}\left(m_{j}\right)$, one for each value of $i$ in $\mathbb{Z}_{d}$.

Similarly, for any two distinct $j, j^{\prime}$ in $J$, the corresponding subgroups $T_{j}$ and $T_{j^{\prime}}$ move disjoint sets of vertices (from disjoint sets of layers of $X$ ), and hence commute with each other, and it follows that the subgroup $N$ generated by all the $T_{j}$ (for $j \in J$ ) is a direct product $\Pi_{j \in J} T_{j} \cong \Pi_{j \in J}\left(S_{m_{j}}\right)^{d_{j}}$, of order $\Pi_{j \in J}\left(m_{j} !\right)^{d_{j}}$.

On the other hand, for fixed $s_{1}$ and $s_{2}$ in $\Omega_{j}$, then

$$
\rho^{-1} \lambda_{i, s_{1}, s_{2}} \rho=\lambda_{i+1, s_{1}, s_{2}} \quad \text { and } \quad \tau^{-1} \lambda_{i, s_{1}, s_{2}} \tau=\lambda_{-i, s_{1}, s_{2}} \quad \text { for all } i \in \mathbb{Z}_{d},
$$

so the automorphisms $\lambda_{i, s_{1}, s_{2}}$ are permuted among themselves in a cycle under conjugation by the rotation $\rho$, and fixed or interchanged in pairs under conjugation by the reflection $\tau$. In particular,

$$
\rho^{-1} T_{j, i} \rho=T_{j, i+1} \quad \text { and } \quad \tau^{-1} T_{j, i} \tau=T_{-j, i} \quad \text { for all } i \in \mathbb{Z}_{d} \text { and all } j \in J .
$$

Finally if $a \in B \backslash\{ \pm 1\}$, and $j^{\prime}$ is the element of (the underlying set of) $J$ congruent to $\pm a j \bmod n$, then the automorphism $\sigma_{a}$ defined in Proposition 9 takes the layers $L_{s}$ for $s \in \Omega_{j}$ to the layers $L_{s^{\prime}}$ for $s^{\prime} \in \Omega_{j^{\prime}}$, and conjugates the subgroup $T_{j}$ (generated by those $\lambda_{i, s_{1}, s_{2}}$ with $s_{1}, s_{2}$ in $\Omega_{j}$ ) to the corresponding subgroup $T_{j^{\prime}}$. Hence $\sigma_{a}$ normalises the subgroup $N=\Pi_{j \in J} T_{j}$.

Thus $N$ is normalised by $\rho$ and $\tau\left(=\sigma_{-1}\right)$ and all the other $\sigma_{a}$, and is therefore normal in $\operatorname{Aut}(X)$. It follows that

$$
A(n ; J)=\langle N \cup\{\rho\} \cup S\rangle \cong N \rtimes\langle\rho\rangle \rtimes S \cong \prod_{j \in J}\left(S_{m_{j}}\right)^{d_{j}} \rtimes C_{n} \rtimes B,
$$


of order

$$
F(n ; J)=n|B| \prod_{j \in J}\left(m_{j} !\right)^{d_{j}},
$$

where the products are taken over all $j$ from the underlying set of $J$, without multiplicities.

For example, in the case of $G I(15 ; 3,5,5,5,6,6)$, the automorphism group has three orbits on vertices, of lengths 15,30 and 45, which are the unions of one, two and three layers (corresponding to the $j$-values 3,6 and 5, respectively). In particular, this graph is not vertex-transitive. Also we have $T_{5} \cong \operatorname{Sym}(3)^{5}$ and $T_{6} \cong \operatorname{Sym}(2)^{3}$ while $T_{3}$ is trivial, and so $F(15 ; 3,5,5,5,6,6)=15 \cdot 4 \cdot\left(1 \cdot 6^{5} \cdot 2^{3}\right)=3732480$.

\subsection{Summary}

Combining the results from the four subsections above gives an algorithm for computing the automorphism groups of $G I$-graphs and their automorphism groups in general.

\section{Vertex-transitive $G I$-graphs}

In this section we consider further symmetry properties of GI-graphs. By Corollary 6, we know there are only eight different connected edge-transitive $G I$-graphs $G I(n ; J)$ having two or more layers. In particular, there are no such graphs with four or more layers. In contrast, we will show that there are several vertex-transitive $G I$-graphs, by giving a classification of them.

Note that the graph $G I(n ; J)$ will be vertex-transitive if we are able to permute the layers of $G I(n ; J)$ transitively among themselves. Now for each non-zero $a \in \mathbb{Z}_{n}$, consider multiplication of the (multi)set $J \cup-J$ by $a$. If this preserves $J \cup-J$ (as a multiset), then it gives a bijection from $J \cup-J$ to $J \cup-J$, and so by Proposition 9, an automorphism $\sigma_{a}$ of $G I(n ; J)$, permuting the layers. The graph $G I(n ; J)$ will be vertex-transitive if the group generated by all such $\sigma_{a}$ acts transitively on the layers.

Theorem 16 Let $J$ be any subset of $\mathbb{Z}_{n}^{*}$ with the two properties that (a) $J \cap-J=\emptyset$, and (b) $J \cup-J$ is a (multiplicative) subgroup of $\mathbb{Z}_{n}^{*}$. Then $G I(n ; J)$ is vertextransitive.

Proof Since $J \cup-J$ is a subgroup of $\mathbb{Z}_{n}^{*}$ (not containing 0 ), multiplication by any $a \in$ $J$ gives an automorphism $\sigma_{a}$ of $G I(n ; J)$ that permutes layers, by our observations above. Moreover, for any $a, b \in J$ there exists $c \in J$ such that $a c= \pm b$ in $\mathbb{Z}_{n}$, and in this case, the automorphism $\sigma_{c}$ takes any layer $s$ with $j_{s}=a$ to a layer $s^{\prime}$ with $j_{s^{\prime}}= \pm b$. It follows that the group generated by $\left\{\sigma_{a}: a \in J\right\}$ acts transitively on the layers of $G I(n ; J)$, and hence the group generated by $\{\rho\} \cup\left\{\sigma_{a}: a \in J\right\}$ acts transitively on the vertices of $G I(n ; J)$.

Corollary 17 Let $A$ be any subgroup of the multiplicative group $\mathbb{Z}_{n}^{*}$ containing an element of $\mathbb{Z}_{n} \backslash\{ \pm 1\}$. If $-1 \in A$, then take $J=A \cap\left\{1,2, \ldots,\left\lfloor\frac{n-1}{2}\right\rfloor\right\}$ (so that $A=$ 


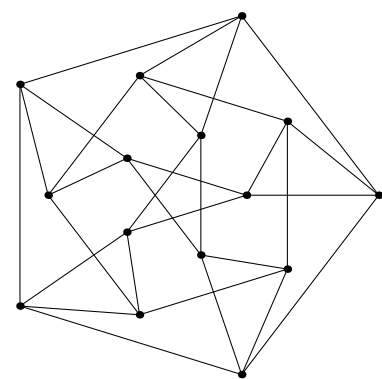

(a)

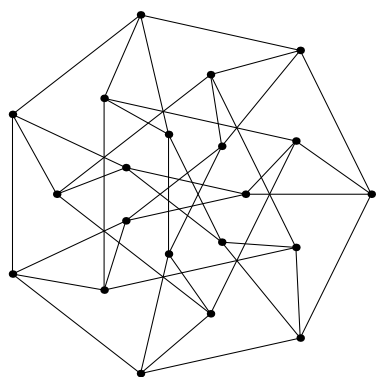

(b)

Fig. 2 The graph $\operatorname{GI}(5 ; 1,1,2)$ in (a) has 5-cycles as its three layers but is not vertex-transitive, while the graph $G I(7 ; 1,2,3)$ in $(\mathbf{b})$ is vertex-transitive and has two edge orbits

$J \cup-J)$, while if $-1 \notin A$, let $J=A$. Then $G I(n ; J)$ is vertex-transitive. Hence for every integer $n>6$, there exists at least one vertex-transitive GI-graph of the form $G I(n ; J)$ for some $J$ with $|J|>1$.

Note that the above requires $\phi(n)=\left|\mathbb{Z}_{n}^{*}\right|$ to be at least 4 , so that $n>4$ and $n \neq 6$, in order for there to be at least two layers. A sub-family consists of those for which $A$ is the cyclic subgroup $\left\{1, r, r^{2}, \ldots, r^{t-1}\right\}$ generated by the powers of a single unit $r \in \mathbb{Z}_{n}^{*} \backslash\{ \pm 1\}$. An example is given in Fig. 2(b), with $n=7$ and $r=2$ (and $2^{2} \equiv 4 \equiv$ $-3 \bmod 7)$.

Next, we say that a subset $J=\left\{j_{0}, j_{1}, \ldots, j_{t-1}\right\}$ of $\mathbb{Z}_{n}$ is primitive if $1 \in J$ and $j_{i} \neq \pm j_{k}$ whenever $i \neq k$. Also we say that the graph $G I(n ; J)$ is primitive if $J$ is a primitive subset of $\mathbb{Z}_{n}$. Note that any such graph is connected, since $1 \in J$.

Theorem 18 A primitive GI-graph $G I(n ; J)$ is vertex-transitive if and only if either $J \cup-J$ is a (multiplicative) subgroup of $\mathbb{Z}_{n}^{*}$, or $n=10$ and $J=\{1,2\}$.

Proof First, it was shown in [6] that $G I(10 ; 1,2)$ is vertex-transitive. Also by Theorem 16 , we know that $G I(n ; J)$ is vertex-transitive when $J \cup-J$ is a subgroup of $\mathbb{Z}_{n}^{*}$.

Conversely, suppose that $X=G I(n ; J)$ is a primitive vertex-transitive $G I$-graph, other than $G I(10 ; 1,2)$. We have to show that $J \cup-J$ is a subgroup of $\mathbb{Z}_{n}^{*}$.

Since $X$ is primitive, we have $1 \in J$, and without loss of generality we may assume that $j_{0}=1$. By Theorem 5, we know that if $X$ has a skew automorphism, then either $t=2$ and $\left(n, j_{1}\right)=(4,1),(5,2),(8,3),(10,2),(10,3),(12,5)$ or $(24,5)$, or $t=3$ and $\left(n, j_{1}, j_{2}\right)=(3,1,1)$. It is easy to see that $J \cup-J$ is a subgroup of $\mathbb{Z}_{n}^{*}$ in all of these cases except $\left(n, t, j_{0}, j_{1}\right)=(10,2,1,2)$. Hence we may assume that $X$ has no skew automorphism, and therefore every automorphism of $X$ preserves the fundamental edge-partition.

Now because $X$ is vertex-transitive, and the layer $L_{0}$ is a single $n$-cycle, it follows that all the layers of $X$ must be cycles, and so every element of $J$ must be coprime to $n$, and therefore a unit $\bmod n$. In particular, there are no automorphisms that 'mix' cycles from different layers. In fact, since $J$ is primitive, $J \cup-J$ contains $2 t$ distinct 
elements, and it follows that $X$ has no automorphisms of the form given in Proposition 7 or Corollary 8.

Hence (by Theorem 12) the only automorphisms that preserve the spoke $S_{0}$ are the automorphisms $\sigma_{a}$ given in Corollary 15.

But for any $x \in J$ (say $x=j_{s}$ ), by vertex-transitivity there exists an automorphism of $X$ that maps the vertex $(0,0)$ to the vertex $(s, 0)$, and this must be one of the automorphisms $\sigma_{a}$, where $a$ is a unit in $\mathbb{Z}_{n}$ and $a(J \cup-J)=J \cup-J$. In particular, since $\sigma_{a}$ takes $(0, v)$ to $(\alpha(0), a v)$ for all $v \in \mathbb{Z}_{n}$, we have $\alpha(0)=s$ and therefore $x=$ $j_{s}=j_{\alpha(0)}= \pm a j_{0}= \pm a$, which gives $x(J \cup-J)= \pm a(J \cup-J)= \pm(J \cup-J)=$ $J \cup-J$, for every $x \in J$.

Thus $J \cup-J$ is closed under multiplication, and by finiteness (and the fact that every element of $J$ is a unit $\bmod n$ ), it follows that $J \cup-J$ is a subgroup of $\mathbb{Z}_{n}^{*}$, as required.

We will now find some other examples, and show that every vertex-transitive $G I$ graph has a special form. To do that, we introduce some more notation: we denote by $[k] J$ the concatenation of $k$ copies of the multiset $J$. Note that this may involve a non-standard ordering of the elements of $[k] J$, but it makes the proofs of some things in this and the next section easier to explain-specifically, Theorem 19 and Lemmas 21 and 22.

Theorem 19 Let $X=G I(n ; J)$ be any connected vertex-transitive GI-graph. Then

(a) If $\operatorname{Aut}(X)$ has a vertex-transitive subgroup that preserves the fundamental edgepartition of $X$, then $G I(n ;[k] J)$ is vertex-transitive for every positive integer $k$.

(b) All elements in $J \cup-J$ have the same multiplicity, say $k_{0}$, and (so conversely) the graph $X=G I(n ; J)$ is isomorphic to $G I\left(n ;\left[k_{0}\right] J_{0}\right)$ for some primitive subset $J_{0}$ of $\mathbb{Z}_{n}$, such that $G I\left(n ; J_{0}\right)$ is vertex-transitive.

Proof Let $X=G I(n ; J)=G I\left(n ; j_{0}, j_{1}, \ldots, j_{t-1}\right)$, and let $Y=G I(n ;[k] J)$.

Note that the vertex set of $Y$ is $\mathbb{Z}_{k t} \times \mathbb{Z}_{n}$, and we can write $[k] J=\left(j_{0}, j_{1}, \ldots\right.$, $j_{k t-1}$ ), where $j_{c}=j_{d}$ whenever $c \equiv d \bmod t$, and accordingly, we can write each member $s$ of $\mathbb{Z}_{k t}$ in the form $a t+b$ where (by a small abuse of notation) $a \in$ $\{0,1, \ldots, k-1\}=\mathbb{Z}_{k}$ and $b \in\{0,1, \ldots, t-1\}=\mathbb{Z}_{t}$.

Also note that any permutation $f$ of $\mathbb{Z}_{k}=\{0,1,2, \ldots, k-1\}$ gives rise to a corresponding permutation $\widetilde{f}$ of $\mathbb{Z}_{k t}=\{0,1,2, \ldots, k t-1\}$, defined (loosely) by setting $\widetilde{f}(a t+b)=f(a) t+b$ for all $a \in \mathbb{Z}_{k}$ and all $b \in \mathbb{Z}_{t}$, and in fact gives rise to an automorphism $\theta=\theta_{f}$ of $Y=G I(n ;[k] J)$, defined by

$\theta_{f}(a t+b, v)=(\tilde{f}(a t+b), v)=(f(a) t+b, v) \quad$ for all $a \in \mathbb{Z}_{k}, b \in \mathbb{Z}_{t}$ and $v \in \mathbb{Z}_{n}$.

It is easy to see that $\theta_{f}$ preserves the edges of each spoke $S_{v}$, and permutes the layers among themselves. In fact $\theta_{f}$ takes $L_{a t+b}$ to $L_{f(a) t+b}$ for all $a \in \mathbb{Z}_{k}$ and all $b \in \mathbb{Z}_{t}$, and hence $\theta_{f}$ preserves each of the sets $\left\{L_{s}: s \in \mathbb{Z}_{k t} \mid s \equiv b \bmod t\right\}$ for $b \in \mathbb{Z}_{t}$.

It follows that given any two layers $L_{c}=\left\{(c, v): v \in \mathbb{Z}_{n}\right\}$ and $L_{d}=\{(d, v)$ : $\left.v \in \mathbb{Z}_{n}\right\}$ with $c \equiv d \bmod t$, there exists an automorphism $\theta$ of $Y$ taking $L_{c}$ to $L_{d}$. 
In particular, since $\operatorname{Aut}(Y)$ is transitive on vertices of each layer (as is the automorphism group of every $G I$-graph), we find that $\operatorname{Aut}(Y)$ has at most $t$ orbits on vertices of $Y$.

We can now prove (a), by extending certain automorphisms of $X$ to automorphisms of $Y$ that make it vertex-transitive.

Let $\xi$ be any automorphism of $X$ that respects the fundamental edge-partition. Define a permutation $\pi=\pi_{\xi}$ of the vertex set of $Y$ by letting

$$
\pi(a t+b, v)=(a t+c, w) \quad \text { whenever } \xi(b, v)=(c, w),
$$

for all $a \in \mathbb{Z}_{k}$, all $b \in \mathbb{Z}_{t}$, and all $v \in \mathbb{Z}_{n}$.

If $e$ is a spoke edge of $Y$, say from $(a t+b, v)$ to $\left(a^{\prime} t+b^{\prime}, v\right)$, and $(c, w)=\xi(b, v)$, then since $\xi$ takes spoke edges to spoke edges in $X$, we see that $\xi\left(b^{\prime}, v\right)=\left(c^{\prime}, w\right)$ for some $c^{\prime} \in \mathbb{Z}_{t}$, and so by definition $\pi\left(a^{\prime} t+b^{\prime}, v\right)=\left(a^{\prime} t+c^{\prime}, w\right)$, which is a neighbour of $(a t+c, w)$. Thus $\pi$ takes the edge $e$ to the spoke edge in $Y$ from $(a t+c, w)$ to $\left(a^{\prime} t+c^{\prime}, w\right)$.

Similarly, if $e$ is a layer edge of $Y$, say from $(a t+b, v)$ to $(a t+b, z)$, with $z=$ $v+j_{b}\left(\right.$ since $j_{d}=j_{d^{\prime}}$ whenever $\left.d \equiv d^{\prime} \bmod t\right)$, and $(c, w)=\xi(b, v)$, then since $\xi$ permutes the layers of $X$, we know that $\xi$ takes the neighbour $(b, z)=\left(b, v+j_{b}\right)$ of $(b, v)$ on the same layer of $X$ as $(b, v)$ to a neighbour of $(c, w)$ on the same layer of $X$ as $(c, w)$, namely $\left(c, w \pm j_{c}\right)$. Hence by definition, $\pi(a t+b, z)=\left(a t+c, w \pm j_{c}\right)$, which is a neighbour of $(a t+c, w)$ in $Y$ because $j_{a t+c}=j_{c}$. Thus $\pi$ takes $e$ to a layer edge from $(a t+c, w)$ to $\left(a t+c, w \pm j_{c}\right)$ in $Y$.

In particular, since $\pi$ preserves both the set of all spoke edges of $Y$ and the set of all layer edges of $Y$, we find that $\pi=\pi_{\xi}$ is an automorphism of $Y$.

Moreover, since $\xi$ can be chosen to take any layer of $X$ to any other layer of $X$, it follows that the subgroup of $\operatorname{Aut}(Y)$ generated by the automorphisms $\theta_{f}$ and $\pi_{\xi}$ found above is transitive on layers of $Y$, and hence $Y$ is vertex-transitive.

Next we prove (b), namely that all elements in $J \cup-J$ have the same multiplicity, say $k_{0}$, and $X$ is isomorphic to $G I\left(n ;\left[k_{0}\right] J_{0}\right)$ for some primitive subset $J_{0}$ of $\mathbb{Z}_{n}$ such that $G I\left(n ; J_{0}\right)$ is vertex-transitive.

If $X$ is edge-transitive, then by Theorem 5 we have $(n ; J)=(4 ; 1,1),(5 ; 1,2)$, $(8 ; 1,3),(10 ; 1,2),(10 ; 1,3),(12 ; 1,5),(24 ; 1,5)$ or $(3 ; 1,1,1)$. In the first case, we can take $k_{0}=2$ and $J_{0}=\{1\}$, and observe that $G I\left(n ; J_{0}\right)=G I(4 ; 1)$ which is simply a 4-cycle, and vertex-transitive. Similarly, in the last case, we can take $k_{0}=3$ and $J_{0}=\{1\}$, and observe that $G I\left(n ; J_{0}\right)=G I(3 ; 1)$ which is a 3 -cycle, and vertextransitive. In all the other six cases, we can take $k_{0}=1$ and $J_{0}=J$, and note that $X=$ $G I(n ; J)$ itself is vertex-transitive. Thus (b) holds in all eight cases, and so from now on, we may assume that $X$ is not edge-transitive, and hence that every automorphism of $X$ respects the fundamental edge-partition.

This implies that $\operatorname{Aut}(X)$ is transitive on the layers of $X$, and it follows that all the layer cycles have the same length, so $\operatorname{gcd}\left(n, j_{s}\right)=\operatorname{gcd}\left(n, j_{0}\right)$ for all $s \in \mathbb{Z}_{t}$. But on the other hand, $X=G I(n ; J)$ is connected, $\operatorname{so} \operatorname{gcd}\left(n, j_{0}, j_{1} \ldots, j_{t-1}\right)=1$. Thus $\operatorname{gcd}\left(n, j_{s}\right)=1$ for all $s \in \mathbb{Z}_{t}$.

In particular, there exists $a \in \mathbb{Z}_{n}^{*}$ such that $1=a j_{s} \in a J$. Now by Proposition 1 , the graph $X=G I(n ; J)$ is isomorphic to $G I(n ; a J)$, and therefore we can replace $J$ by $a J$, or more simply, suppose that $1 \in J$. 
If all the elements of $J$ are the same, then $X=G I(n ; J)$ is isomorphic to $G I(n ;[t]\{1\})$, and then since the set $\{1\}$ is primitive and $G I(n ; 1)$ is simply an $n$ cycle, again (b) holds.

So now suppose that not all elements of $J$ are the same. For any two distinct $j_{i}, j_{s} \in J$, there must be an automorphism $\sigma_{a}$ that takes layer $L_{i}$ to layer $L_{s}$, by Corollary 15. In this case $a(J \cup-J)=J \cup-J$, by definition of $\sigma_{a}$, and therefore the multiplicities of $j_{i}$ and $j_{s}$ are the same. Hence all elements of $J \cup-J$ have the same multiplicity, say $k_{0}$.

In particular, $J=\left[k_{0}\right] J_{0}$ where $J_{0}$ is the underlying set of $J$, and $X$ is isomorphic to $G I\left(n ;\left[k_{0}\right] J_{0}\right)$. The set $J_{0}$ is primitive since it contains 1 and all of its elements are distinct. To finish the proof, all we have to do is show that $G I\left(n ; J_{0}\right)$ is vertextransitive. But that is easy: for any two distinct $j_{i}, j_{s} \in J_{0}$, we know that there exists an automorphism $\sigma_{a}$ of $X$ taking layer $L_{i}$ of $X$ to layer $L_{s}$ of $X$, and $a(J \cup-J)=$ $J \cup-J$; it then follows that $a\left(J_{0} \cup-J_{0}\right)=J_{0} \cup-J_{0}$, and therefore $\sigma_{a}$ induces an automorphism of $G I\left(n ; J_{0}\right)$ that takes layer $L_{i}$ of $G I\left(n ; J_{0}\right)$ to layer $L_{s}$ of $G I\left(n ; J_{0}\right)$, as required.

Note that the above theorem applies only to connected $G I$-graphs. Disconnected vertex-transitive $G I$-graphs are just disjoint unions of connected vertex-transitive $G I$ graphs, and can be dealt with accordingly.

We finish this section with observations about the graphs $\operatorname{GI}(5 ; 1,2)$ and $G I(10 ; 1,2)$.

The Petersen graph $G I(5 ; 1,2)$ is vertex-transitive, and its automorphism group acts transitively on the two layers; in fact so does a subgroup of order 20 which preserves the set of its ten layer edges. By Theorem 19, it follows that every $G I$-graph of the form $G I(5 ; 1,2,1,2, \ldots, 1,2)$ is vertex-transitive.

On the other hand, the automorphism group of the dodecahedral graph $G I(10 ; 1,2)$ has no layer-transitive subgroup preserving the set of layer edges (and the set of spoke edges), and so the above theorem does not apply to it. In fact $G I(10 ;[k]\{1,2\})$ is not vertex-transitive for any $k>1$, because the fact that 2 is not a unit mod 10 implies that the automorphism group has two orbits on layers.

The graph $G I(10 ; 1,2)$ is the only such exception, since for every other vertextransitive $G I$-graph $X$, either $\operatorname{Aut}(X)$ itself preserves the fundamental edge-partition, or $X$ is edge-transitive and is then one of the other seven graphs in Theorem 5, and for each of those, the subgroup of $\operatorname{Aut}(X)$ preserving the fundamental edge-partition is layer-transitive.

\section{Cayley GI-graphs}

In this section we characterize the $G I$-graphs that are Cayley graphs.

First, a Cayley graph $\operatorname{Cay}(G, S)$ is a graph whose vertices can be labelled with the elements of some group $G$, and whose edges correspond to multiplication by the elements of some subset $S$ or their inverses. In particular, the edges of $\operatorname{Cay}(G, S)$ may be taken as the pairs $\{g, g s\}$ for all $g \in G$ and all $s \in S$, and then the group $G$ acts naturally as a group of automorphisms of $\operatorname{Cay}(G, S)$ by left multiplication. This 
action is transitive on vertices, indeed regular on vertices: for any ordered pair $(u, v)$ of vertices, there is a unique element of $G$ taking $u$ to $v$ (namely $g=v^{-1} u$ ).

Alternatively, a Cayley graph is any (regular) graph $X$ whose automorphism group has a subgroup $G$ that acts regularly on vertices. In that case, any particular vertex can be labelled with the identity element of $G$, and the subset $S$ can be taken as the set of all $s \in G$ taking that vertex to one of its neighbours.

Note that under both definitions, the Cayley graph is connected if and only if the set $S$ generates the group $G$. Also note that every Cayley graph is vertex-transitive (by definition), and that every non-trivial element of the subgroup $G$ fixes no vertices of the graph.

Now suppose $X=G I(n ; J)$ is a vertex-transitive $G I$-graph.

We will assume that $X$ is connected, because if it is not, then it is simply a disjoint union of isomorphic copies of a connected smaller example. In particular, by Theorem 19, we know that either $J$ is primitive (and $X$ is one of the graphs given by Theorem 18), or all elements in $J \cup-J$ have the same multiplicity $k_{0}>1$ and then $X$ is isomorphic to $G I\left(n ;\left[k_{0}\right] J_{0}\right)$ for some primitive subset $J_{0}$ of $\mathbb{Z}_{n}$ such that $G I\left(n ; J_{0}\right)$ is vertex-transitive.

Also we will suppose that $X$ is not $G I(10 ; 1,2)$, for reasons related to Theorem 18 . In fact, of the seven generalized Petersen graphs among the eight edge-transitive GI-graphs listed in Theorem 5, it is known by the main result of [18] or [14] that $G(4,1), G(8,3), G(12,5)$ and $G(24,5)$ are Cayley graphs, while $G(5,2), G(10,2)$ and $G(10,3)$ are not. Most of this (and the fact that the eighth edge-transitive graph $G I(3 ; 1,1,1)$ is a Cayley graph) will actually follow from what we prove below.

Consider the case where $J$ is primitive (as we defined in Sect. 5). In this case, $J \cup-J$ is a subgroup of $\mathbb{Z}_{n}^{*}$ under multiplication, and also $|\operatorname{Aut}(X)|=n|J \cup-J|=$ $2 n|J|=2|V(X)|$.

Hence if $G$ is a subgroup of $\operatorname{Aut}(X)$ that acts regularly on vertices of $X$, then $G$ is a subgroup of index 2 in $\operatorname{Aut}(X)$. On the other hand, $G$ cannot contain the element $\tau$, since $\tau$ is a non-trivial automorphism with fixed points (namely the vertices $(s, 0)$ for all $s$ ), and it follows that $G$ must be generated by the rotation $\rho$ and some subgroup of index 2 in $\left\{\sigma_{a}: a \in J \cup-J\right\}$ not containing $\sigma_{-1}=\tau$. The latter has to be of the form $\left\{\sigma_{a}: a \in K\right\}$ for some subgroup $K$ of $J \cup-J$, such that $-1 \notin K$.

Conversely, if $J$ is a set, and $K$ is a subgroup of index 2 in $J \cup-J$ not containing -1 , then the group generated by $\left\{\sigma_{a}: a \in K\right\}$ permutes the layers of $X$ transitively, and so the subgroup generated by $\{\rho\} \cup\left\{\sigma_{a}: a \in K\right\}$ acts regularly on $V(X)$.

Thus we have the following:

Proposition 20 If $G I(n ; J)$ is primitive and $J \cup-J$ is a multiplicative subgroup of $\mathbb{Z}_{n}^{*}$, then $G I(n ; J)$ is a Cayley graph if and only if $J \cup-J$ has a subgroup of index 2 that does not contain -1 .

Note that this gives infinitely many examples of GI-graphs that are Cayley graphs, including those where $n$ is a prime congruent to $3 \bmod 4$ and $J$ is the subgroup of all squares in $\mathbb{Z}_{n}^{*}$. On the other hand, it also gives infinitely many vertex-transitive $G I$-graphs that are not Cayley graphs, including those where $n$ is a prime congruent to $1 \bmod 4$ and $J \cup-J=\mathbb{Z}_{n}^{*}=\mathbb{Z}_{n} \backslash\{0\}$. 
This proposition also shows that among the six primitive $G I$-graphs that are edge-transitive, $G I(8 ; 1,3), G I(12 ; 1,5)$ and $G I(24 ; 1,5)$ are Cayley graphs, while $G I(5 ; 1,2)$ and $G I(10 ; 1,3)$ are not. (The graph $G I(10 ; 1,2)$ is not a Cayley graph, for other reasons.)

Next, consider the more general case, where $X=G I(n ; J)$ is connected and vertex-transitive. In this case, by Theorem 19, we know that all elements in $J \cup-J$ have the same multiplicity $k_{0}$, and $X$ is isomorphic to $G I\left(n ;\left[k_{0}\right] J_{0}\right)$ for some primitive subset $J_{0}$ of $\mathbb{Z}_{n}$, such that $G I\left(n ; J_{0}\right)$ is vertex-transitive. Also by what we found in Sect. 5 and Sect. 4.4, we have $d_{j}:=\operatorname{gcd}(n, j)=1$ for all $j \in J_{0}$, and therefore

$$
|\operatorname{Aut}(X)|=n\left|J_{0} \cup-J_{0}\right| \prod_{j \in J_{0}}\left(k_{0} !\right)^{d_{j}}=2 n\left|J_{0}\right|\left(k_{0} !\right)^{\left|J_{0}\right|} .
$$

We will find the following helpful, and to state it, we will refer to the automorphism $\rho$ of each $G I$-graph $G I(n ; J)$ as its standard rotation, and sometimes denote it by $\rho_{J}$.

Lemma 21 If $G I(n ; J)$ has a vertex-regular subgroup containing the standard rotation, then so does $G I(n ;[k] J)$ for every integer $k>1$.

Proof Let $X=G I(n ; J)$ and $Y=G I(n ;[k] J)$, and let $\rho\left(=\rho_{J}\right)$ be the standard rotation for $X$. Also let $\{\rho\} \cup S$ be a generating set for a vertex-regular subgroup of $\operatorname{Aut}(X)$. Note that $\operatorname{Aut}(X)$ is layer-transitive on $X$, since $X$ is not $G I(10 ; 1,2)$. Now by multiplying elements of $S$ by powers of $\rho$ if necessary, we may assume that $\langle S\rangle$ induces a regular permutation group on the set of layers of $X$. In particular, $\langle S\rangle$ has order $|J|$. Next, for each $\xi \in S$, the automorphism $\pi_{\xi}$ defined in the proof of Theorem 19 acts fixed-point-freely on $Y=G I(n ;[k] J)$, and it follows that the set $\left\{\pi_{\xi}: \xi \in S\right\}$ generates a subgroup of order $|J|$ that permutes the layers of $Y=G I(n ;[k] J)$ in $|J|$ blocks of size $k$. Also if $f$ is the $k$-cycle $f=(1,2, \ldots, k)$ in $\operatorname{Sym}(k)$, then the automorphism $\theta_{f}$ defined in the proof of Theorem 19 induces a $k$-cycle on each of those $|J|$ layer-blocks. Finally, $\theta_{f}$ commutes with $\rho_{[k] J}$ and all the $\pi_{\xi}$ (for $\xi \in S$ ), so the subgroup generated by $\rho_{[k] J}, \theta_{f}$ and all the $\pi_{\xi}$ has order $n k|J|$, and acts regularly on the vertices of $Y$, as required.

Note that this shows, for example, that both of the remaining two edge-transitive $G I$-graphs $G I(4 ; 1,1)$ and $G I(3 ; 1,1,1)$ are Cayley graphs.

Somewhat surprisingly, we also have the following:

Lemma 22 If $J$ is primitive and both $G I(n ; J)$ and $G I(n ;[2] J)$ are vertex-transitive, then $G I(n ;[2] J)$ is always a Cayley graph, and so is $G I(n ;[k] J)$ for every even integer $k>1$.

Proof First, if $G I(n ; J)$ is a Cayley graph, then this follows from Lemma 21, so we will assume that $G I(n ; J)$ is not a Cayley graph. Also because $G I(n ;[2] J)$ is vertextransitive, we know that $G I(n ; J) \neq G I(10 ; 1,2)$, and so $J \cup-J$ is a subgroup of $\mathbb{Z}_{n}^{*}$, by Theorem 18. On the other hand, by Proposition 20, we know that $J \cup-J$ has no subgroup of index 2 that excludes -1 . Hence we can write $J \cup-J$ as $U \times W$, where 
$U$ is a cyclic 2-subgroup containing -1 and of order $q=2^{e}$ for some $e>1$, and $W$ is complementary to $U$, and of order $2 t / q$. Also let $u$ be a generator of $U$, so that $u^{q / 2}=-1$.

Now consider the automorphisms of $Y=G I(n ;[2] J)$. For each $a \in J \cup-J=$ $U \times W$, without loss of generality we will choose the associated bijection $\alpha: \mathbb{Z}_{2 t} \rightarrow \mathbb{Z}_{2 t}$ to be the 'duplicate' of the corresponding natural bijection from $\mathbb{Z}_{t}$ to $\mathbb{Z}_{t}$, namely so that $\alpha$ takes $s$ to $s^{\prime}$, and $s+t$ to $s^{\prime}+t$, whenever $j_{s^{\prime}}=j_{s^{\prime}+t}=$ $\pm a j_{s}= \pm a j_{s+t}$ (for $0 \leq s<t$ ).

For the moment, suppose that $W$ is trivial, so that $U=J \cup-J$. Then the automorphism $\sigma_{u}$ is not semi-regular, because the vertex $(0,0)$ lies in a cycle of length $q / 2$ consisting of all $(s, 0)$ with $0 \leq s<t$ and $\pm j_{s}=u^{i}$ for some $i$, while the vertex $(0,1)$ lies in a cycle of length $q$ consisting of all $\left(s, u^{i}\right)$ such that $0 \leq s<t$ and $\pm j_{s}=u^{i}$, for $0 \leq i<q$. Hence in particular, the subgroup generated by $\rho$ and $\sigma_{u}$ has order $n q=2 n t$, but cannot be vertex-regular (since the $(q / 2)$ th power of $\sigma_{u}$ is a non-trivial element with fixed points).

On the other hand, we can multiply $\sigma_{u}$ by $\lambda_{0, t}$, which interchanges vertices $(0, v)$ and $(t, v)$, for all $v \in \mathbb{Z}_{n}$, and find that $\sigma_{u} \lambda_{0, t}$ is a semi-regular element of order $q$, with $n / q$ cycles of length $q$. (The vertex $(0,0)$ lies in a cycle of length $q=2 t$ consisting of all $\left(s^{\prime}, 0\right)$ with $\pm j_{s^{\prime}}=u^{i}$ for some $i$, while the vertex $(0,1)$ lies in a cycle of length $q$ consisting of all $\left(s, u^{i}\right)$ such that $0 \leq s<t$ and $\pm j_{s}=u^{i}$ for even $i$, and all $\left(s+t, u^{i}\right)$ such that $0 \leq s<t$ and $\pm j_{s}=u^{i}$ for odd $i$; the cycles containing the other vertices $\left(s^{\prime}, 1\right)$ have a similar form.)

It follows that the subgroup generated by $\rho$ and $\sigma_{u} \lambda_{0, t}$ has order $n q=2 n t$, and is transitive on vertices, and hence is vertex-regular, so that $G I(n ;[2] J)$ is a Cayley graph.

When $W$ is non-trivial, the elements $\sigma_{w}$ for all $w$ in $W$ (or simply all $w$ from a generating set for $W$ ) induce a regular permutation group on the layers $L_{s}$ for which $\pm j_{s} \in W$, and it follows that the subgroup generated by $\rho$ and $\sigma_{u} \lambda_{0, t}$ and these $\sigma_{w}$ acts regularly on the vertices of $Y$, again making $G I(n ;[2] J)$ a Cayley graph.

Finally, for any even integer $k>2$, we find that $G I(n ;[k] J)=G I(n ;[k / 2][2] J)$ is a Cayley graph, by applying Lemma 21 with [2] $J$ in place of $J$, and $k / 2$ in place of $k$.

On the other hand, the same kind of thing does not occur when $k$ is odd:

Lemma 23 If $J$ is primitive and $G I(n ; J)$ is vertex-transitive but not a Cayley graph, then $G I(n ;[k] J)$ is not a Cayley graph for any odd integer $k>1$.

Proof Assume the contrary, so that $X=G I(n ; J)$ is vertex-transitive and not a Cayley graph, but $Y=G I(n ;[k] J)$ is a Cayley graph, for some odd $k$.

Then we know that $X \neq G I(10 ; 1,2)$, since $Y$ is vertex-transitive, and so $J \cup-J$ is a subgroup of $\mathbb{Z}_{n}^{*}$, by Theorem 18 . On the other hand, since $X$ is not a Cayley graph, Proposition 20 tells us that $J \cup-J$ has no subgroup of index 2 that excludes -1 , and therefore $J \cup-J$ contains an element $u$ of (multiplicative) order $4 m$ for some $m$, with $u^{2 m}=-1$. Also by Theorem 5, we know that $Y$ is not edge-transitive, and so $\operatorname{Aut}(Y)$ preserves the fundamental edge-partition of $Y$, and hence every subgroup of $\operatorname{Aut}(Y)$ permutes the layers of $Y$ among themselves. 
Now let $R$ be a vertex-regular subgroup of $\operatorname{Aut}(Y)$, and take $b=u^{m}$, which has order 4 , with $b^{2}=-1$ in $\mathbb{Z}_{n}^{*}$. Next, choose $i$ such that $j_{i}= \pm b$ (noting that such an $i$ must exist because $b$ lies in the subgroup $J \cup-J$ ). Then by vertex-transitivity of $R$, there exists some automorphism $\theta$ of $Y$ taking the vertex $(0,0)$ to the vertex $(i, 0)$. Moreover, by our knowledge of the structure of $\operatorname{Aut}(Y)$ from Sect. 4 and the fact that all of the automorphisms $\lambda_{s_{1}, s_{2}}$ and $\sigma_{a}$ preserve the spoke $S_{0}$, it follows that $\theta=w \sigma_{b}$ or $w \sigma_{-b}$ for some $w$ in the subgroup $N$ generated by the set of all of the automorphisms $\lambda_{s_{1}, s_{2}}$.

Since $R$ acts regularly on vertices, every non-trivial automorphism in $R$ has to be semi-regular. In particular, $\theta$ is semi-regular, as is its square

$$
\theta^{2}=\left(w \sigma_{ \pm b}\right)^{2}=w\left(\sigma_{ \pm b} w \sigma_{ \pm b}^{-1}\right) \sigma_{( \pm b)^{2}}=w^{\prime} \sigma_{-1}=w^{\prime} \tau
$$

where $w^{\prime}=w\left(\sigma_{ \pm b} w \sigma_{ \pm b}^{-1}\right) \in N$. Both $w^{\prime}$ and $\tau$ preserve the spoke $S_{0}$, and therefore so does $w^{\prime} \tau$, and thus $w^{\prime} \tau$ acts semi-regularly on $S_{0}$. But also $\tau$ fixes every vertex $(s, 0)$ of $S_{0}$, and so $w^{\prime}$ itself acts semi-regularly on $S_{0}$. Furthermore, since every element of $N$ preserves the set $\left\{L_{0}, L_{t}, \ldots, L_{(k-1) t}\right\}$ of $k$ layers corresponding to the occurrences of 1 in $J$, it follows that both $w^{\prime} \tau$ and $w^{\prime}$ act semi-regularly on the set $K=\{(r t, 0): 0 \leq r<k\}$.

In particular, cycles of the permutation induced by $w^{\prime}$ on $K=\{(r t, 0): 0 \leq r<k\}$ must all have the same length, say $\ell$. Note that $w^{\prime}$ is non-trivial, for otherwise $w^{\prime} \tau=\tau$, which is not semi-regular on vertices (because it has fixed points), and therefore $\ell>1$. But also $\ell$ must divide $k$, so $\ell$ is odd.

Now consider any $\ell$-cycle of $w^{\prime}$ on $K$, say $\left(\left(s_{1}, 0\right),\left(s_{2}, 0\right), \ldots,\left(s_{\ell}, 0\right)\right)$. Because $\tau$ fixes every vertex of $K$, this is also a cycle of $w^{\prime} \tau$, and hence all cycles of $w^{\prime} \tau$ have length $\ell$. Also by definition of the elements generating $N$ (as defined in Proposition 7$)$, we know that $\left(\left(s_{1}, 1\right),\left(s_{2}, 1\right), \ldots,\left(s_{\ell}, 1\right)\right)$ must be a cycle of $w^{\prime}$. But now the cycle of $w^{\prime} \tau$ containing the vertex $\left(s_{1}, 1\right)$ is

$\left(\left(s_{1}, 1\right),\left(s_{2},-1\right),\left(s_{3}, 1\right), \ldots,\left(s_{\ell-1},-1\right),\left(s_{\ell}, 1\right),\left(s_{1},-1\right),\left(s_{2}, 1\right),\left(s_{3},-1\right), \ldots,\left(s_{\ell},-1\right)\right)$,

which has length $2 k$, and this contradicts the fact that $w^{\prime} \tau$ is semi-regular.

Putting together Proposition 20 and Lemmas 21 and 22, we have the following:

Theorem 24 If $X=G I(n ; J)$ is connected, then $X$ is a Cayley graph if and only if

(a) $J$ is primitive, and $J \cup-J$ is a multiplicative subgroup of $\mathbb{Z}_{n}^{*}$, with a subgroup of index 2 that does not contain -1 , or

(b) $X=G I(n ; J)$ is isomorphic to $G I\left(n ;\left[k_{0}\right] J_{0}\right)$ for some primitive subset $J_{0}$ of $\mathbb{Z}_{n}$ and some integer $k_{0}>1$, such that either $G I\left(n ; J_{0}\right)$ is a Cayley graph, or $k_{0}$ is even and $G I\left(n ; J_{0}\right)$ is vertex-transitive but is not the dodecahedral graph $G I(10 ; 1,2)$.

\section{Additional remarks}

The family of $G I$-graphs forms a natural generalization of the Petersen graph. Our initial studies of $G I$-graphs have shown that this family is indeed very interesting 
Fig. 3 The graph $G I(7 ; 1,2,3)$ as a unit-distance graph

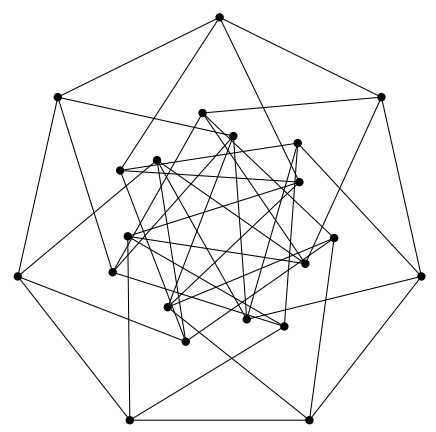

and deserves further consideration. These graphs are also related to circulant graphs [17]. Through that relationship, we were able to solve the puzzle of what appeared to be unstructured automorphisms of $G I$-graphs, and this enabled us to find their automorphism groups and classify those that are vertex-transitive or Cayley graphs. Symmetries of graphs offer additional interesting questions and may open new viewpoints in the study of the structure of graphs. For instance, it would be interesting to investigate consistent cycles of GI-graphs. Consistent cycles were introduced by John H. Conway in a talk in 1971; for a recent reference on this topic, see [16].

Let us mention also the problem of unit-distance drawings of GI-graphs. A graph is a unit-distance graph if it can be drawn in the plane such that all of its edges have the same length. In [10], it was shown that all $I$-graphs are unit-distance graphs. On the other hand, obviously no GI-graph with four or more layers can be a unit-distance graph, since it contains a $K_{4}$ as a subgraph, which itself is not a unit-distance graph. Hence the only open case of interest is the sub-class of $G I$-graphs having three layers.

For each $k \in \mathbb{Z}_{n}$, the graph $G I(n ; k, k, k)$ is a cartesian product of two cycles, or a disjoint union of such products, and is therefore a unit-distance graph, by [9, Theorem 3.4]. We know of only one other connected example that is a unitdistance graph, and it is remarkable.

This is the graph $\operatorname{GI}(7 ; 1,2,3)$, which is shown in Fig. 3.

The vertices can be drawn equidistantly on three concentric circles with radii

$$
R_{1}=\frac{1}{2 \sin (\pi / 7)}, \quad R_{2}=\frac{1}{2 \sin (2 \pi / 7)}, \quad \text { and } \quad R_{3}=\frac{1}{2 \sin (3 \pi / 7)},
$$

and the two smaller circles rotated through angles of $\pi / 3$ and $-\pi / 3$ with respect to the largest circle. One can then verify that all edges have the same length 1.

The graph $G I(7 ; 1,2,3)$ is a Cayley graph for the non-abelian group of order 21 , namely $\mathbb{Z}_{7} \rtimes_{2} \mathbb{Z}_{3}$, which has presentation $\left\langle a, b \mid a^{7}=b^{3}=1, b^{-1} a b=a^{2}\right\rangle$. Its girth is 3 but it contains no cycles of length 4 . This means that its Kronecker cover (see [12]) has girth 6 and is a Levi graph $[3,20]$ of a self-polar, point- and line-transitive but not flag-transitive combinatorial $\left(21_{4}\right)$-configuration. The resulting configuration is different from the configuration of Grünbaum and Rigby [7], since the latter configuration is flag-transitive but the one obtained from $\operatorname{GI}(7 ; 1,2,3)$ is not. 
Acknowledgements This work has been financed by ARRS within the EUROCORES Programme EuroGIGA (project GReGAS, N1-0011) of the European Science Foundation. It was also supported partially by the ARRS (via grant P1-0294), and the N.Z. Marsden Fund (via grant UOA1015).

\section{References}

1. Boben, M., Pisanski, T., Žitnik, A.: I-graphs and the corresponding configurations. J. Comb. Des. 13, 406-424 (2005)

2. Bosma, W., Cannon, J., Playoust, C.: The magma algebra system I: The user language. J. Symb. Comput. 24, 235-265 (1997)

3. Coxeter, H.S.M.: Self-dual configurations and regular graphs. Bull. Am. Math. Soc. 56, 413-455 (1950)

4. Bouwer, I.Z., Chernoff, W.W., Monson, B., Star, Z.: The Foster Census. Charles Babbage Research Centre (1988)

5. Frucht, R.: On the groups of repeated graphs. Bull. Am. Math. Soc. 55, 418-420 (1949)

6. Frucht, R., Graver, J.E., Watkins, M.E.: The groups of the generalized Petersen graphs. Proc. Camb. Philol. Soc. 70, 211-218 (1971)

7. Grünbaum, B., Rigby, J.F.: The real configuration (214). J. Lond. Math. Soc. 41, 336-346 (1990)

8. Harary, F.: Graph Theory. Addison-Wesley, Reading (1969)

9. Horvat, B., Pisanski, T.: Products of unit distance graphs. Discrete Math. 310, 1783-1792 (2010)

10. Horvat, B., Pisanski, T., Žitnik, A.: All generalized Petersen graphs are unit-distance graphs. J. Korean Math. Soc. 49, 475-491 (2012)

11. Horvat, B., Pisanski, T., Žitnik, A.: Isomorphism checking of $I$-graphs. Graphs Comb. 28, 823-830 (2012)

12. Imrich, W., Pisanski, T.: Multiple Kronecker covering graphs. Eur. J. Comb. 29, 1116-1122 (2008)

13. Lovrečič Saražin, M., Pacco, W., Previtali, A.: Generalizing the generalized Petersen graphs. Discrete Math. 307, 534-543 (2007)

14. Lovrečič Saražin, M.: A note on the generalized Petersen graphs that are also Cayley graphs. J. Comb. Theory, Ser. B 69, 226-229 (1997)

15. Lovrečič Saražin, M., Marušič, D.: Vertex-transitive expansions of (1, 3)-trees. Discrete Math. 310, 1772-1782 (2010)

16. Miklavič, Š.: A note on a conjecture on consistent cycles. Ars Math. Contemp. 6, 389-392 (2013)

17. Morris, J.: Automorphisms of circulants that respect partitions. Submitted. arXiv:1205.0503v1

18. Nedela, R., Škoviera, M.: Which generalized Petersen graphs are Cayley graphs? J. Graph Theory 19, 1-11 (1995)

19. Petkovšek, M., Zakrajšek, H.: Enumeration of I-graphs: Burnside does it again. Ars Math. Contemp. 2, 241-262 (2009)

20. Pisanski, T., Servatius, B.: Configurations from a Graphical Viewpoint. Birkhäuser Advanced Texts. Birkhäuser, New York (2013)

21. Watkins, M.: A theorem on Tait colorings with an application to the generalized Petersen graphs. J. Comb. Theory 6, 152-164 (1969) 